\title{
Preparation of a Near-Infrared Ray Absorption Film from $N$-Phenylthiocarbamoyl Chitosan Derivative
}

\author{
Shouko Nishida, Masaya Shibano, Hiroshi Kamitakahara and Toshiyuki Takano* \\ Received: 18 September 2015; Accepted: 27 November 2015; Published: 4 December 2015 \\ Academic Editors: Hitoshi Sashiwa and Shinsuke Ifuku \\ Division of Forest and Biomaterials Science, Graduate School of Agriculture, Kyoto University, \\ Kyoto 606-8502, Japan; s.nishida.kyoto.agriculture@gmail.com (S.N.); ma.shibano@gmail.com (M.S.); \\ hkamitan@kais.kyoto-u.ac.jp (H.K.) \\ * Correspondence: takatmys@kais.kyoto-u.ac.jp; Tel.: +81-75-753-6254; Fax: +81-75-753-6300
}

\begin{abstract}
We recently observed that the decanoylation of $N$-phenylthiocarbamoyl chitosan (2) with a mixture of decanoic anhydride and pyridine at $60{ }^{\circ} \mathrm{C}$ for $24 \mathrm{~h}$ afforded $\mathrm{N}, \mathrm{N}$-(decanoyl)phenythiocarbamoyl-/2-isothiocynato chitosan decanoate $(3 \mathbf{b})$ rather than the expected product $\mathrm{N}, \mathrm{N}$-(decanoyl)phenylthiocarbamoyl chitosan decanoate (3a). This result suggested that some of the $\mathrm{N}, \mathrm{N}$-(decanoyl)phenylthiocarmbamoyl groups had been converted to isothiocyanate groups during the decanoylation process. The subsequent reaction of compound $\mathbf{3 b}$ with aniline gave $N, N-($ decanoyl)phenylthiocarbamoyl $/ N$-phenylthiocarbamoyl chitosan decanoate (4) in high yield. A solution of compound 4 in $\mathrm{CHCl}_{3}$ was then added to a solution of copper decanoate (5) in the same solvent, and the resulting mixture was cast onto a glass plate to give a cast film. The film was annealed at $200{ }^{\circ} \mathrm{C}$ in an oven to give a greenish film, which showed good near-infrared absorption characteristic in the range of $800-2200 \mathrm{~nm}$.
\end{abstract}

Keywords: chitosan; copper; decanoylation; near-infrared absorption; phenythiocarbamoyl

\section{Introduction}

Chitosan is derived from the $\mathrm{N}$-deacetylation of chitin, which is the second most abundant of the known natural biopolymers. Although a strict nomenclature has not yet been defined with respect to the degree of the $\mathrm{N}$-deacetylation of chitosan, chitin with the degree of $\mathrm{N}$-deacetylation of more than $40 \%$ is generally considered to be chitosan. Numerous chitosan derivatives have been reported for a wide range of interesting applications, including foods, cosmetics, and pharmaceuticals [1-3]. Despite these advances, considerable research interests are still being focused on the development of new high-value added applications for chitosan and its derivatives. $N$-Substituted thiocarbamoyl chitosan derivatives are one of the important groups of functional chitosan derivatives to have been reported to date. For example, $\mathrm{N}$-acetyl-, $\mathrm{N}$-fluoresceinyl-, and $\mathrm{N}$-phenyl-thiocarbamoyl chitosan derivatives have been reported to behave as antimicrobial materials [4], corrosion inhibitors [5], macromolecular fluorophores [6], and metal adsorbents [7,8].

Near-infrared rays are defined as light in the range of 780-2500 nm. Approximately half of the solar energy emitted from the sun caused by near-infrared rays $[9,10]$. It is therefore envisaged that materials capable of adsorbing near-infrared rays could be used as heat-ray shielding films to prevent a temperature rise in a room and in a car for housing and car windows during the summer season [10], as well as being used as heat-ray absorption films during the winter season and heat storage fibers for winter clothes. The absorbing materials are also expected as optical filters for a plasma display panel [11] and as near-infrared bio-imaging materials [12]. We previously reported that a powder mixture of $\mathrm{N}$-phenylthiocarbamoyl chitosan and copper stearate could be heated at 200-250 ${ }^{\circ} \mathrm{C}$ to afford a near-infrared absorption material [13]. However, our subsequent investigation 
of the absorption capabilities of this material revealed that they were inadequate. For example, the degree of substitution with $N$-phenylthiocarbamoyl groups (DSPhNHCS) of the chitosan derivatives prepared in this way was low (0.33) and the powder mixture could not be converted to a cast film because it was not soluble in common organic solvents. However, we recently reported the development of a synthetic method for preparation of $N$-phenylthiocarbamoyl chitosan with a much higher DSphNHCS value of 0.85 [11]. Notably, we prepared $N, N$-(acyl)phenylthiocarbamoyl chitosan acylate from this material and found that it showed good properties as a film for the adsorption of near-infrared rays.

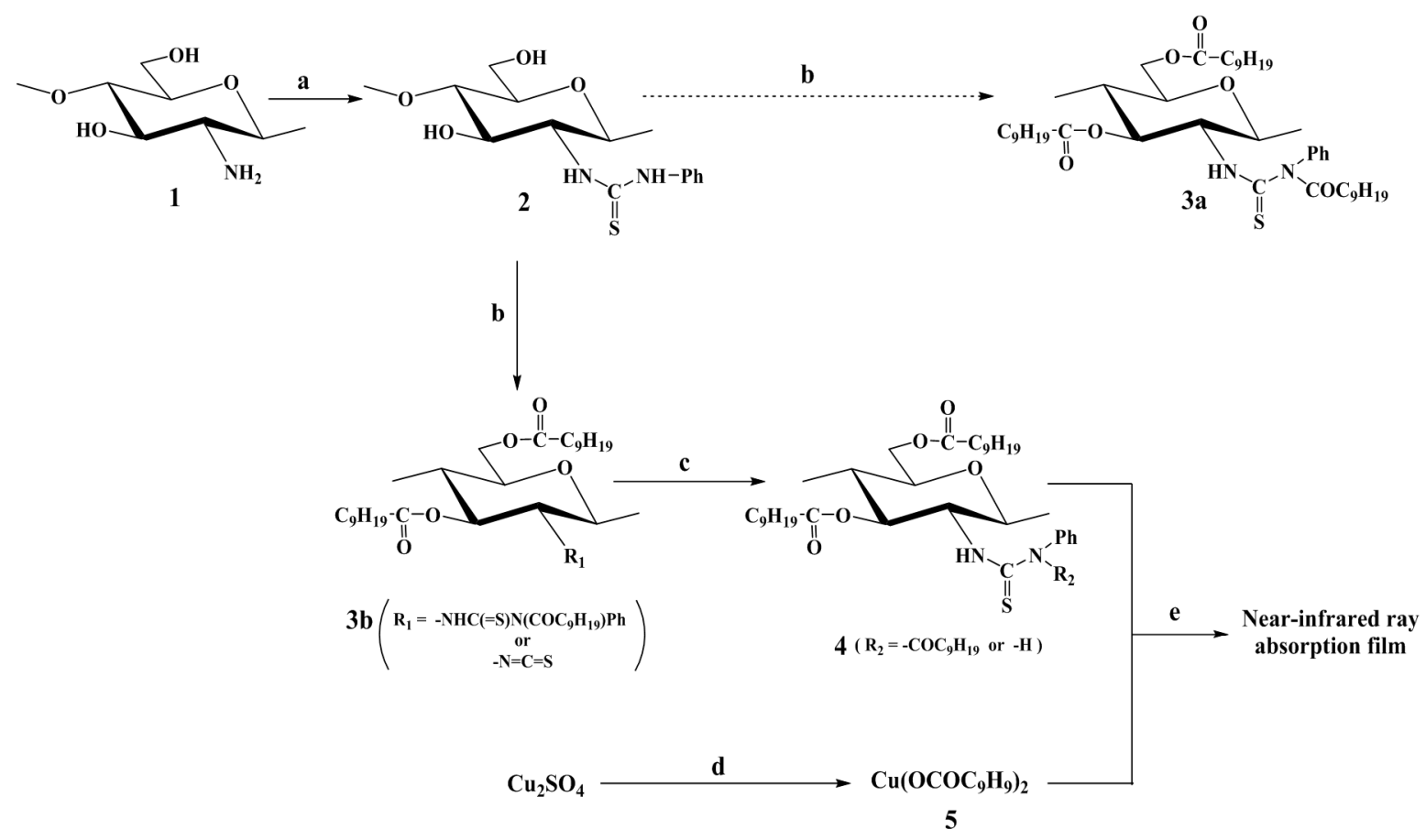

(a) $\mathrm{Ph}=\mathrm{N}=\mathrm{C}=\mathrm{S} / 5 \%-\mathrm{AcOH} / \mathrm{H}_{2} \mathrm{O}, 35^{\circ} \mathrm{C}, 24 \mathrm{~h}$; (b) $\mathrm{C}_{9} \mathrm{H}_{19} \mathrm{COC} 1 /$ pyridine, $60{ }^{\circ} \mathrm{C}, 24 \mathrm{~h}$; (c) $\mathrm{C}_{6} \mathrm{H}_{5} \mathrm{NH}_{2} / \mathrm{CHCl}_{3}, 60^{\circ} \mathrm{C}$, $24 \mathrm{~h} ;(\mathbf{d}) \mathrm{C}_{9} \mathrm{H}_{9} \mathrm{COOH} / 30 \%$-ammonium solution/petroleum ether, r.t., $30 \mathrm{~min}$; and (e) annealing at $200{ }^{\circ} \mathrm{C}$.

Scheme 1. Preparation of a near-infrared ray absorption film.

In this work, $N, N$-(decanoyl)phenylthiocarbamoyl chitosan decanoate (3a) was initially selected as a promising chitosan derivative for the development of a film for the adsorption of near-infrared rays. This material was selected as a suitable candidate because the results of a preliminary experiment had shown that the introduction of $n$-decanoyl groups at the $0-3$ and $O-6$ positions of chitosan led to an increase in its solubility in several common organic solvents. Surprisingly, however, this process resulted in the preparation of $\mathrm{N}, \mathrm{N}$-(decanoyl)phenylthiocarbamoyl/ $\mathrm{N}$-phenylthiocarbamoyl chitosan decanoate (4) instead of compound 3a (Scheme 1). This paper describes the preparation of compound 4 from chitosan (1) and an evaluation of its behavior as a film for the adsorption of near-infrared rays.

\section{Results and Discussion}

\subsection{Preparation of $\mathrm{N}, \mathrm{N}-($ Decanoyl)phenylthiocarbamoyl Chitosan Decanoate (4)}

The decanoylation of compound 2 [14] with a mixture of decanoic anhydride in pyridine at $60{ }^{\circ} \mathrm{C}$ for $24 \mathrm{~h}$ afforded product (A) in high yield. The FT-IR spectrum of this material contained characteristic ester and amide bands at 1740 and $1676 \mathrm{~cm}^{-1}$, respectively, whereas the bands attributed to the hydroxyl groups of the starting material around $3298 \mathrm{~cm}^{-1}$ had been reduced significantly (Figure 1). NMR analysis of product (A) revealed the characteristic signals of the decanoyl groups at 2.35-0.89 ppm and 33.9-14.1 ppm in its ${ }^{1} \mathrm{H}$ and ${ }^{13} \mathrm{C}$ NMR spectra, respectively. Taken together, these 
results suggested that the $\mathrm{O}$ - and $\mathrm{N}$-decanoylation reactions had proceeded smoothly at the $\mathrm{OH}-3$, $\mathrm{OH}-6$, and $\mathrm{N}$-phenylthiocarbamoyl groups of the starting material. Notably, the FT-IR and ${ }^{13} \mathrm{C}$ NMR spectra of product (A) contained a band at $2046 \mathrm{~cm}^{-1}$ and a signal at $141.8 \mathrm{ppm}$, respectively, which were attributed to the isothiocyanate (NCS) groups [14]. These results suggested that the isothiocyanation reaction had proceeded during the acylation of compound 2 with a mixture of acyl anhydride in pyridine. This result was similar to that reported in our previous paper using an acyl halide/pyridine system [15] and confirmed that product (A) was compound $\mathbf{3 b}$ rather than $\mathbf{3 a}$.

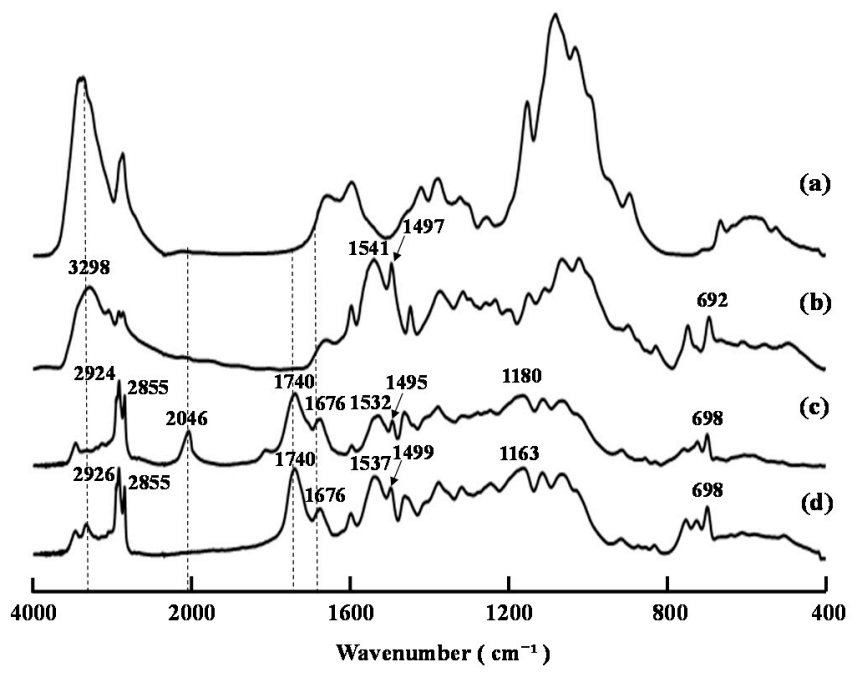

Figure 1. FT-IR spectra of compounds (a) 1, (b) 2, (c) 3b (product (A)), and (d) 4 .

Figure 2 shows the FT-IR spectra of the products resulting from the decanoylation of compound 2 at various time intervals when the reaction temperature was $60^{\circ} \mathrm{C}$. The characteristic bands of the amide groups at $1678 \mathrm{~cm}^{-1}$ rapidly appeared within only $1 \mathrm{~h}$, and then gradually decreased, whereas the band belonging to the NCS groups at $2046 \mathrm{~cm}^{-1}$ gradually increased with increasing reaction time. This result showed that the formation of the NCS groups was caused by the degradation of the resulting $\mathrm{N}, \mathrm{N}$-(decanoyl)phenylthiocarbamoyl groups during the $\mathrm{O}$-decanoylation. A small band of NCS groups at $2045 \mathrm{~cm}^{-1}$ could be detected after only $1 \mathrm{~h}$, suggesting that the formation of NCS groups was unavoidable when the reaction temperature was set as $60^{\circ} \mathrm{C}$.

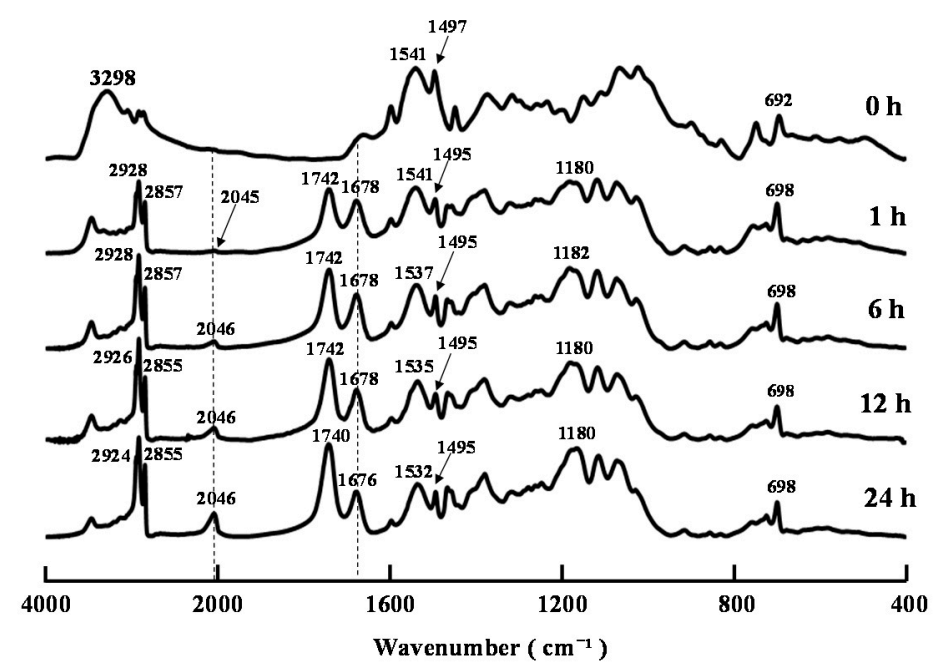

Figure 2. FT-IR spectra of decanoylation products of compound 2 after 0-24 h. 
Wet compound $\mathbf{3 b}$ was reacted with aniline in $\mathrm{CHCl}_{3}$ to afford compound $\mathbf{4}$ in high yield. It is noteworthy that acyl chitosan isothiocyanates became insoluble in several solvents, including tetrahydrofuran (THF), $\mathrm{CHCl}_{3}$, and $\mathrm{CH}_{2} \mathrm{Cl}_{2}$ when they are stored in the presence of a drying solid at ambient temperature for more than a week [14]. The characteristic NCS band at $2046 \mathrm{~cm}^{-1}$ was found to be absent from the FT-IR spectrum of compound 4, which suggested that this material was $\mathrm{N}, \mathrm{N}$-(decanoyl) phenylthiocarbamoyl/N-phenylthiocarbamoyl chitosan decanoate. The total DS value of $\mathrm{N}, \mathrm{N}$-(decanoyl) phenylthiocarbamoyl and $\mathrm{N}$-phenylthiocarbamoyl groups (DSphenylthiocarbamoyls) for compound 4 was determined to be 0.82 by elemental analysis. The solubility characteristics of compounds 2 and 4 are summarized in Table 1. Compound 4 was found to be soluble in THF, $\mathrm{CHCl}_{3}$, acetone, $\mathrm{CH}_{2} \mathrm{Cl}_{2}$, 1,4-dioxane and DMF, but insoluble in DMSO, $\mathrm{MeOH}$, and $\mathrm{H}_{2} \mathrm{O}$.

Table 1. Solubility of compounds 2 and 4.

\begin{tabular}{cccccccccc}
\hline Solvents & THF & $\mathrm{CHCl}_{3}$ & Acetone & $\mathrm{CH}_{2} \mathrm{Cl}_{2}$ & Dioxane & DMF & DMSO & $\mathrm{CH}_{3} \mathrm{OH}$ & $\mathrm{H}_{2} \mathrm{O}$ \\
\hline$\delta$ & 9.1 & 9.3 & 9.4 & 9.6 & 9.8 & 11.5 & 12.8 & 12.9 & 21.0 \\
Compound 2 & $x$ & $x$ & $x$ & $x$ & $x$ & $\mathrm{O}$ & $\mathrm{O}$ & $\mathrm{x}$ & $\mathrm{x}$ \\
Compound 4 & $\mathrm{O}$ & $\mathrm{O}$ & $\mathrm{O}$ & $\mathrm{O}$ & $\mathrm{O}$ & $\mathrm{O}$ & $\mathrm{x}$ & $\mathrm{x}$ & $\mathrm{x}$ \\
\hline
\end{tabular}

$\delta$ : Solubility parameter; O: Soluble; $x$ : Insoluble.

\subsection{Preparation of Copper(II) Decanoate (5)}

Compound 5 was prepared from decanoic acid and copper sulfate according to a modified version of a reported method for the preparation of copper stearate [16]. Figure 3 shows the FT-IR spectra of decanoic acid and compound 5. The characteristic bands of the carboxyl groups in decanoic acid at 1711 and $1685 \mathrm{~cm}^{-1}$ clearly shifted to 1597 and $1582 \mathrm{~cm}^{-1}$, and the band of the hydroxyl groups at $935 \mathrm{~cm}^{-1}$ disappeared in the FT-IR spectrum of compound 5 . The results were now consistent with the presence of carboxylate groups, which suggested that copper(II) decanoate had been successfully formed. Compound 5 was found to be readily soluble in $\mathrm{THF}, \mathrm{CHCl}_{3}$, and $\mathrm{CH}_{2} \mathrm{Cl}_{2}$.

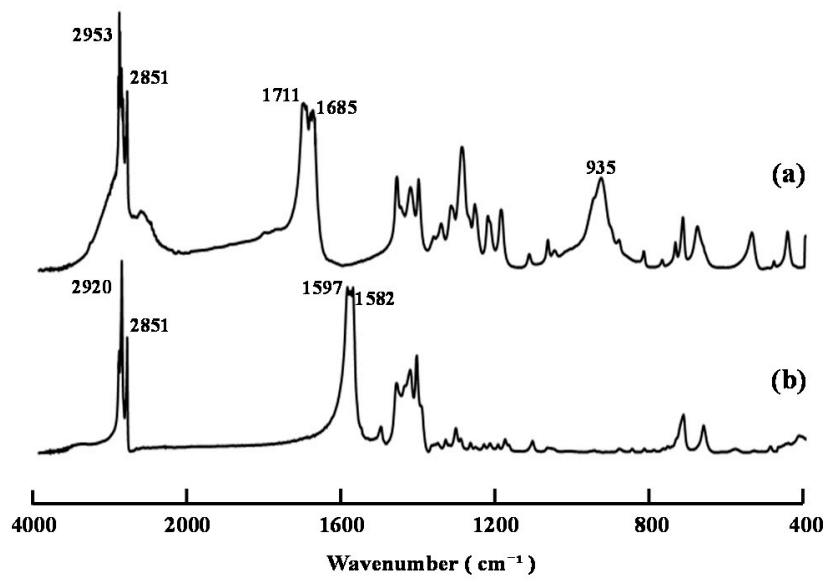

Figure 3. FT-IR spectra of (a) decanoic acid and (b) compound 5.

\subsection{Preparation and Characterization of a Cast Film from the Chitosan Derivative 4 and Copper Decanoate (5)}

It has been reported that an annealing temperature of $200-250{ }^{\circ} \mathrm{C}$ is essential to allow for the formation of materials with near-infrared ray absorption capabilities from a powdered mixture of $N$-phenylthiocarbamoyl chitosan and copper stearate [13]. With this in mind, we investigated the optimum conditions required to achieve the formation of materials with good near-infrared ray absorption capabilities. We initially subjected a $1: 1(\mathrm{~mol} / \mathrm{mol})$ mixture of compounds 4 and 5 to thermogravimetric analysis (TGA) to determine the annealing temperature. The results of this analysis revealed that the thermal degradation of this mixture began at $152{ }^{\circ} \mathrm{C}$, and proceeded 
smoothly thereafter (Figure 4). The weight percent losses at 200 and $250{ }^{\circ} \mathrm{C}$ were $10.7 \%$ and $31.5 \%$, respectively. Based on this result, the optimum annealing temperature was determined to be $200{ }^{\circ} \mathrm{C}$.

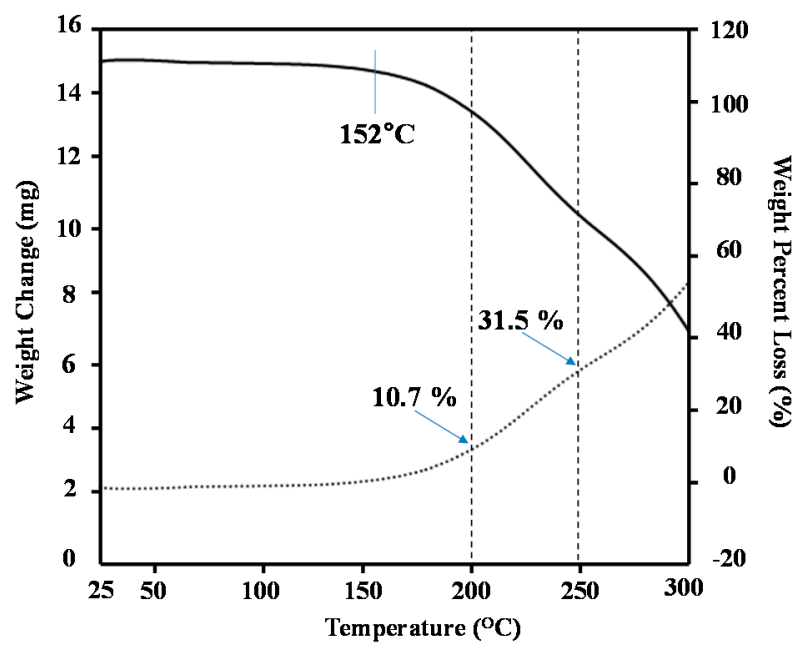

Figure 4. TGA of powder mixture of compounds $\mathbf{4}$ and 5 with a molar ratio $=1: 1$.

Cast films were subsequently constructed from a 1:1 (mol/mol) mixture of compounds 4 and 5 on glass substrates and annealed at $200{ }^{\circ} \mathrm{C}$ for different times in an oven. Figure 5 shows the UV-visible-NIR transmittance spectra of the films treated for different annealing times. The transmittance of light through the films in the range of $800-2200 \mathrm{~nm}$ was found to be $100 \%$ before the annealing process, which showed that these films possessed no near-infrared ray absorption capability. The transmittance of light in the near-infrared region through the films decreased after an annealing time of $1 \mathrm{~min}$ (Figure 5), which indicated that the near-infrared ray absorption ability of the films was increasing with increased annealing time. The level of transmittance in the near-infrared ray region decreased again as the annealing time was increased from 1 to $3 \mathrm{~min}$, but increased only slightly when the annealing time was increased from 3 to $5 \mathrm{~min}$. The transmittance of the film after an annealing time of $3 \mathrm{~min}$ was $56.9 \%$ at $1166 \mathrm{~nm}$ (at the minimum transmittance wavelength). Based on these results, the optimum annealing time was determined to be $3 \mathrm{~min}$.

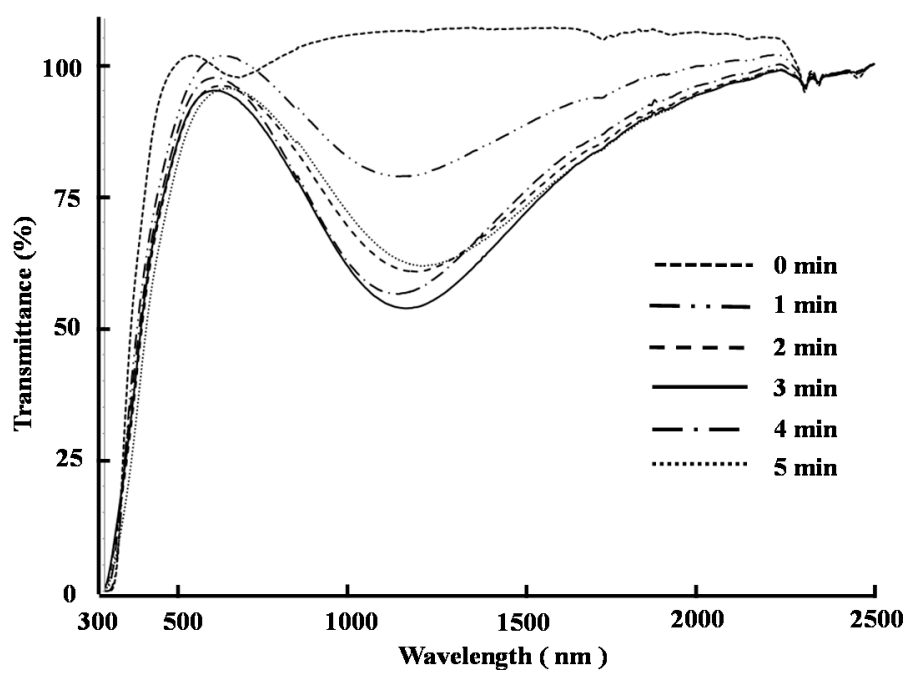

Figure 5. UV-visible-NIR transmittance spectra of films of compounds 4 and 5 with a molar ratio $=1: 1$ after the heating treatment at $200^{\circ} \mathrm{C}$ for $0,1,2,3,4$, and $5 \mathrm{~min}$.

We then investigated the constructed of a variety of different films using different molar ratios of compounds 4 and 5, which were prepared and treated at $200{ }^{\circ} \mathrm{C}$ for $3 \mathrm{~min}$ in an oven. Figure 6 shows the UV-visible-NIR transmittance spectra of these films after they had been subjected to the 
optimal annealing conditions described above. These results revealed that the level of transmittance in the near-infrared region decreased as the ratio of compounds 4 and 5 increased from 1:1 to 1:5, but then increased when the molar ration was increased from 1:5 to 1:6. The reason for this red-shift in the minimum transmittance wavelength of the film with a molar ratio of 1:6 is not yet clear. Based on these results, the optimum molar ratio of compounds 4 to 5 was determined to be 1:5.

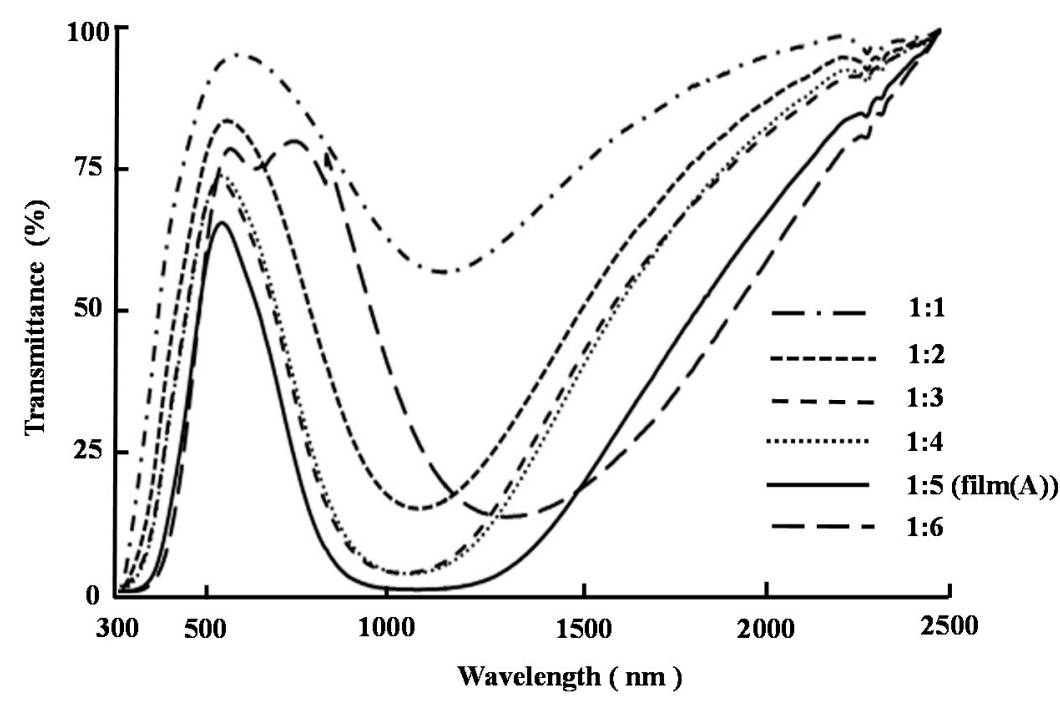

Figure 6. UV-visible-NIR transmittance spectra of films with the molar ratio of compounds 4 and $5=1: 1,1: 2,1: 3,1: 4,1: 5$, and 1:6 after heating treatment at $200^{\circ} \mathrm{C}$ for $3 \mathrm{~min}$.

Taken together, the results of the optimization experiments described above showed that films prepared from a 1:5 ( $\mathrm{mol} / \mathrm{mol})$ ratio of compounds 4 and 5 after an annealing time of $3 \mathrm{~min}$ at $200{ }^{\circ} \mathrm{C}$ (i.e., film (A)) showed the best near-infrared ray absorption ability (Figure 6). Film (A) was found to be greenish in color, as shown in Figure 7 . The transmittance of film (A) was less than $1 \%$ at wavelengths in the range of 978-1216 nm, which suggested that film (A) possessed excellent near-infrared absorption capabilities. However, the transmittance characteristics of film (A) at wavelengths in the range of 300-750 $\mathrm{nm}$ (at visible ray region) decreased compared with how they were before the annealing process. Indeed, the transmittance at $568 \mathrm{~nm}$ (at the maximum transmittance wavelength at visible ray region) was $65.6 \%$.

\section{Before annealing}

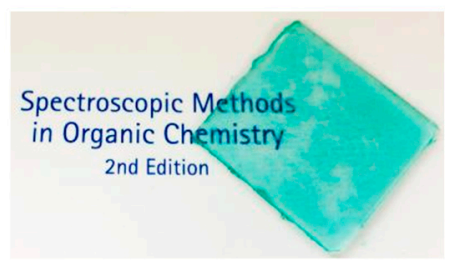

\section{After annealing}

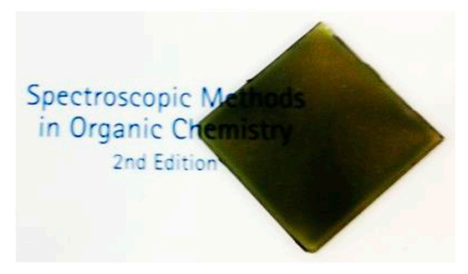

Figure 7. Photograph of film (A) (compounds 4:5=1:5) before and after annealing.

One of the possible reasons for the generation of the near-infrared ray absorption capability of film (A) could be the formation of micro particles of copper sulfide. It is noteworthy that the UV-visible-NIR transmittance spectrum of film (A) was similar to that of a copper sulfide thin film 
prepared from copper(II) chloride and thiourea using a chemical bath method followed by a period of annealing at $300{ }^{\circ} \mathrm{C}[17,18]$. It has also been reported that copper(II) ions can promote the desulfurization of $\mathrm{N}$-phenylthiourea [19]. With this in mind, we evaluated film (A) both before and after the annealing process by FE-SEM and FT-IR spectroscopy. Unfortunately, we only observed a small number of micro particles in film (A) after the annealing process, and it was therefore not possible for this film to adsorb near-infrared rays. Figure 8 shows the FT-IR spectra of compounds 4 and 5 and film (A) before and after the annealing process. The FT-IR spectrum of film (A) before the annealing process (Figure 8c) revealed that there had been a significant decrease in the characteristic carboxylate band of compound 5 at 1597 and $1582 \mathrm{~cm}^{-1}$, and that the characteristic ester band of compound 4 at $1740 \mathrm{~cm}^{-1}$ had shifted to $1697 \mathrm{~cm}^{-1}$. This spectrum also showed that the bands derived from the $N$-phenythiocarbamoyl groups of compound 4 at 1676, 1537, and $1499 \mathrm{~cm}^{-1}$ [14] had disappeared, even though the molar ratio of compound 4 in film (A) was low. It has been reported that 3,6-di-O-decanoyl chitosan dithiocarbamate [20] and $\mathrm{N}$-phenylthiocarbamoyl chitosan [7] can absorb copper(II) ions with a high level of selectivity. These results therefore suggested that the copper(II) ions had coordinated to the decanoyl and $N$-phenylthiocarbamoyl groups of compound 4 rather than the decanoic acid groups of compound $\mathbf{5}$ during the preparation of film (A) before the annealing process. The ester band at $1740 \mathrm{~cm}^{-1}$ and the bands from the $N$-phenythiocarbamoyl groups at 1655, 1544, and $1500 \mathrm{~cm}^{-1}$ appeared in the FT-IR spectrum of film (A) after the annealing process, which suggested the decomposition of the copper ions coordinated to compound 4 and the possibility of the formation of carbon sulfide. However, further investigations would be required to prove this hypothesis.

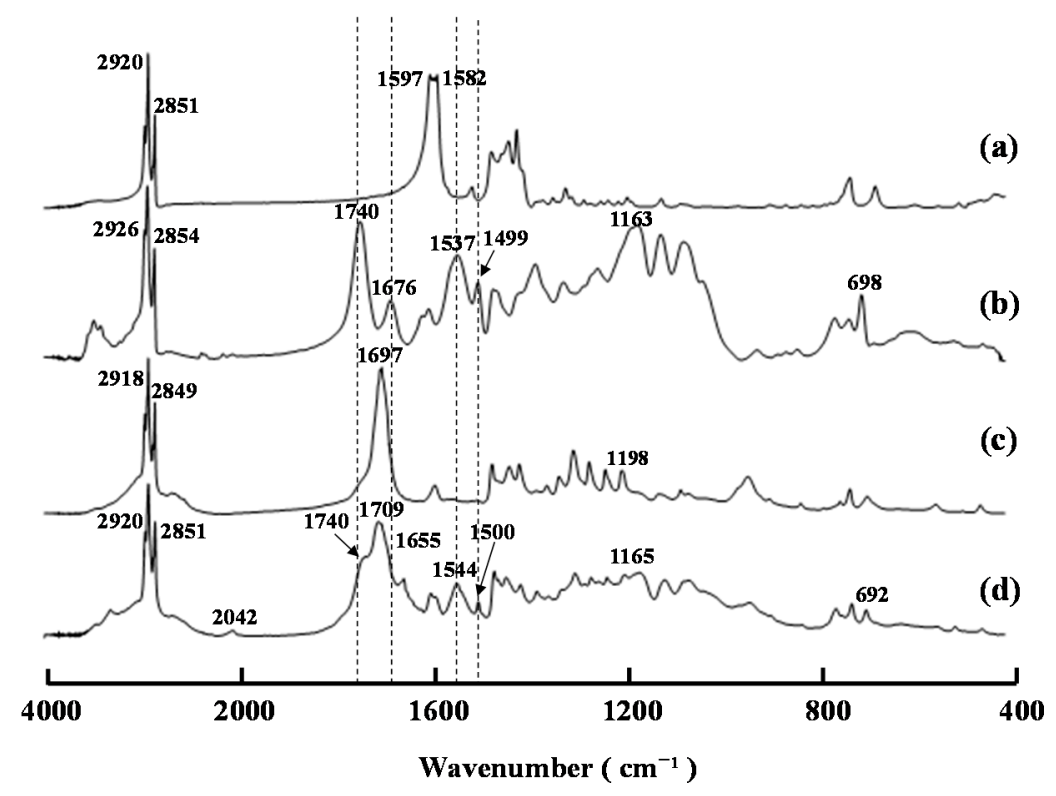

Figure 8. FT-IR spectra of (a) compound 5; (b) compound 4; (c) film A before the heating treatment; and (d) film $\mathbf{A}$ after the heating treatment.

\section{Experimental Section}

\subsection{General}

Chitosan (DAICHITOSAN 100D (VL), degree of deacetylation: 98\%) (1) was kindly supplied by the Dainichiseika Color and Chemicals Manufacturing Company (Tokyo, Japan). $N$-Phenylthiocarbamoyl chitosan (2) with a DSPhNHCS value of 0.85 was prepared from chitosan (1) according to a previously reported procedure [14]. All the other chemicals used in the study were purchased from commercial sources and used without further purification.

Fourier-transform-infrared (FT-IR) spectra were recorded on a Shimadzu IR Prestige-21 spectrophotometer (Shimadzu, Kyoto, Japan) as KBr pellets (1 mg of sample/200 mg of $\mathrm{KBr}$ ). ${ }^{1} \mathrm{H}$ and 
${ }^{13} \mathrm{C}$ NMR spectra were recorded on a Varian FT-NMR (500 MHz) spectrophotometer (Agilent Technologies, Santa Clara, CA, USA) using tetramethylsilane (TMS) as an internal reference standard in $\mathrm{CDCl}_{3}$. The standard number of scans for the ${ }^{1} \mathrm{H}$ and ${ }^{13} \mathrm{C}$ NMR measurements were 3500 and 22,000, respectively. Chemical shifts $(\delta)$ have been reported in parts per million (ppm). Gel permeation chromatography (GPC) was performed on a Shimadzu LC-10 system equipped with a Shimadzu UV-vis detector (SPD-10AVp) and a Shimadzu RI detector (RID-10A) under the following conditions: columns: K-802 + K-802.5 + K-805 in series; column temperature, $40{ }^{\circ} \mathrm{C}$; flow rate, $1.0 \mathrm{~mL} / \mathrm{min}$; standards, polystyrene standards (Shodex, Tokyo, Japan). Thermogravimetric analysis (TGA) was conducted in nitrogen (flow rate: $60 \mathrm{~mL} / \mathrm{min}$ ) with a Shimadzu TGA-50 thermal analyzer. The samples for TGA were heated from 25 to $300{ }^{\circ} \mathrm{C}$ at a programming rate of $15{ }^{\circ} \mathrm{C} / \mathrm{min}$. UV-vis-NIR spectra were recorded on a Jasco V-560 UV-vis spectrophotometer (Jasco, Tokyo, Japan).

\subsection{Preparation of Chitosan Derivative 4 and Copper Decanoate (5)}

\subsubsection{N,N-(Decanoyl)phenylthiocarbamoyl Chitosan Decanoate (4)}

Compound $2(1.02 \mathrm{~g}, 3.38 \mathrm{mmol})$ and decanoic anhydride $(11.0 \mathrm{~g}, 33.8 \mathrm{mmol})$ were added to pyridine $(30 \mathrm{~mL})$, and the resulting suspension was stirred at $60{ }^{\circ} \mathrm{C}$ for $24 \mathrm{~h}$. The reaction was then cooled to ambient temperature and diluted with EtOAc. The organic layer was washed sequentially with a $1 \mathrm{M}$ solution of aqueous $\mathrm{HCl}$, a saturated aqueous solution of $\mathrm{NaHCO}_{3}$ and brine before being dried over anhydrous $\mathrm{Na}_{2} \mathrm{SO}_{4}$. The solvent was then removed under vacuum to afford crude products as an oil. The oil was added in a dropwise manner to $\mathrm{EtOH}(300 \mathrm{~mL})$ with stirring to give a precipitate, which was collected by centrifugation $(20,142 \times g(0 \mathrm{~min})$ and washed twice with EtOH. A small portion of the isolated precipitate was dried under vacuum at ambient temperature for $6 \mathrm{~h}$ and used for the characterization of compound $3 \mathbf{b}(0.682 \mathrm{~g})$, whilst the remainder was used without drying for the preparation of compound 4 .

Aniline $\left(3.1 \mathrm{~mL}, 33.8 \mathrm{mmol}\right.$ ) was added to a solution of the wet precipitate in $\mathrm{CHCl}_{3}(10 \mathrm{~mL})$, and the resulting mixture was stirred at $60{ }^{\circ} \mathrm{C}$ for $24 \mathrm{~h}$. The reaction was then cooled to ambient temperature and poured into $\mathrm{MeOH}(100 \mathrm{~mL})$ to give a precipitate, which was collected by centrifugation $(15,000 \times \mathrm{g}, 10 \mathrm{~min})$ and dissolved in a small amount of EtOAc. The EtOAc solution was then added to $\mathrm{MeOH}(100 \mathrm{~mL})$ in a dropwise manner to give a precipitate, which was collected by centrifugation $(15,000 \times g, 10 \mathrm{~min})$, washed with $\mathrm{MeOH}$ and dried under vacuum at ambient temperature for $6 \mathrm{~h}$ to afford compound 4 as a white solid $(1.43 \mathrm{~g})$.

Compound 3b: FT-IR (KBr) v (cm-1): 3472, 3215, 2924, 2855, 2046, 1740, 1676, 1598, 1532, 1495, 1465, 1379, 1315, 1246, 1180, 1117, 1072, 752, 723, 698. ${ }^{1} \mathrm{H}$ NMR (CDCl $): \delta 12.1$ (NH), 7.53-7.06 (phenyl-H), 5.17 (H-3), 4.48 (H-1, H-6a), 4.27 (H-6b), 3.68 (H-2, H-4, H-5), 2.35, 1.64, 1.27 (decanoyl - $\mathrm{CH}_{2}-$ ), 0.89 (decanoyl $\left.-\mathrm{CH}_{3}\right)$ ppm; ${ }^{13} \mathrm{C}$ NMR $\left(\mathrm{CDCl}_{3}\right)$ : $\delta 186.3(\mathrm{C}=\mathrm{S}), 178.0,173.9,173.3$ (decanoyl $\mathrm{C}=\mathrm{O}$ ), 141.7 (NCS), 129.9, 129.0, 124.2 (phenyl-C), 103.1, 99.7 (C-1), 75.4 (C-4), 73.4 (C-5), 72.3 (C-3), 62.3 (C-6), 60.8, 59.5 (C-2), 34.0, 31.9, 29.3, 24.7, 22.7, (decanoyl - $\mathrm{CH}_{2}-$ ), 14.1 (decanoyl-CH3) ppm.

Compound 4: DSphenylthiocarbamoyls $=0.82$ (by elemental analysis); DPn $=78(\mathrm{Mw} / \mathrm{Mn}=2.56)$; FT-IR (KBr) v (cm-1): 3474, 3339, 2926, 2855, 1740, 1676, 1599, 1537, 1499, 1462, 1377, 1319, 1246, 1163, 1112, 1063, 752, 725, 698. ' ${ }^{\mathrm{H}} \mathrm{NMR}\left(\mathrm{CDCl}_{3}\right):$ $\delta$ 7.66-7.06 (phenyl-H), 5.31-3.48 (H-1,H-2, H-3, H-4, H-5, H-6a, H-6b), 2.35, 1.63, 1.26 (decanoyl $-\mathrm{CH}_{2}-$ ), 0.89 (decanoyl $\left.-\mathrm{CH}_{3}\right) \mathrm{ppm}$; ${ }^{13} \mathrm{C} \mathrm{NMR}\left(\mathrm{CDCl}_{3}\right): \delta 179.3$ (C=S), 173.3, 171.5 (decanoyl C=O), 136.5, 129.8, 124.2 (phenyl-C), 101.1 (C-1), 76.0-68.0 (C-3, C-4, C-5), 62.9 (C-6), 58.2 (C-2), 33.9, 31.9, 29.4, 24.7, 22.7, (decanoyl- $\mathrm{CH}_{2}-$ ), 14.1 (decanoyl-CH3) ppm.

\subsubsection{Preparation of Copper(II) Decanoate (5)}

Distilled water $(3.5 \mathrm{~mL})$ was added to a stirred solution of decanoic acid $(1.40 \mathrm{~g}, 8.13 \mathrm{mmol})$ in petroleum ether $(7 \mathrm{~mL})$, followed by $30 \%$ aqueous ammonium (five drops by a disposal pipette) and a $1 \mathrm{M}$ solution of copper sulfate $(4.7 \mathrm{~mL})$. The resulting mixture was then stirred at ambient temperature for $30 \mathrm{~min}$ before being extracted with $\mathrm{CHCl}_{3}$. The organic layer was then washed four times with distilled water, dried over $\mathrm{Na}_{2} \mathrm{SO}_{4}$ and concentrated under vacuum to give compound 5 as a blue solid.

Compound 5: FT-IR $v\left(\mathrm{~cm}^{-1}\right)$ : 2920, 2851, 1597, 1582, 1467, 1433, 1416, 1315, 1186, 721, 669. 


\subsection{Preparation of a Cast Film from Compounds 4 and 5}

Cover glass $(26 \times 26 \mathrm{~mm})$ was placed at the bottom of an aluminum foil cup with a diameter of $54 \mathrm{~mm}$. A solution of compounds 4 in $\mathrm{CHCl}_{3}\left(8.16 \times 10^{-3} \mathrm{mmol} / \mathrm{mL}, 10 \mathrm{~mL}\right)$ was then treated with a solution of compound 5 in the same solvent at a predetermined molar ratio. The resulting mixture was then stirred at ambient temperature for $15 \mathrm{~min}$ and poured into the aluminum foil cup. The cup was held at ambient temperature for $24 \mathrm{~h}$ and then heated at $200{ }^{\circ} \mathrm{C}$ for a predetermined period of time in an oven. The cup was then removed from the oven and cooled to ambient temperature to give cover glass with the resulting film.

\section{Conclusions}

Target compound 3a was not obtained under the conditions used in the current study because the isothiocyanation reaction proceeded during the decanoylation process. However, compound 4 with a high DS of phenylthiocarbamoyl groups (0.82) was obtained from this decanoylation process following an $N$-phenylthiocarbamoylation reaction. The cast film was prepared from a mixture of compound 4 and copper decanoate 5 in $\mathrm{CHCl}_{3}$ with a molar ratio of 1:5, and further annealed at $200{ }^{\circ} \mathrm{C}$ for $3 \mathrm{~min}$ to give a greenish film, which showed near-infrared absorption performance in the wide range of 800-2200 nm. This film could be easily prepared and be applied as a heat-ray shielding (or absorption) film and provides a further example of the high-value added utilization of chitosan derivatives.

Acknowledgments: The authors are grateful for funding from the A-STEP FS stage Exploratory Research Program (FY2012: AS232Z02373D) from the Japan Science and Technology Agency.

Author Contributions: Shouko Nishida fully contributed to the experiments (synthesis of the chitosan derivatives, preparation of the cast films and evaluation of the near-infrared ray absorption capabilities); Masaya Shibano conducted many preliminary experiments whose outcome can lead to this research; Hiroshi Kamitakahara supported the synthesis and characterization of the chitosan derivatives; and Toshiyuki Takano designed this research and wrote the manuscript.

Conflicts of Interest: The authors declare no conflict of interest.

\section{References}

1. Ravi Kumar, M.N.V.; Muzzarelli, R.A.A.; Muzzarelli, C.; Sashiwa, H.; Domb, A.J. Chitosan chemistry and pharmaceutical perspectives. Chem. Rev. 2004, 104, 6017-6084.

2. Alves, N.M.; Mano, J.F. Chitosan derivatives obtained by chemical modifications for biomedical and environmental applications. Int. J. Biol. Macromol. 2008, 43, 401-414.

3. Sahoo, D.; Sahoo, S.; Mohanty, P.; Sasmal, S.; Nayak, P.L. Chitosan: A new versatile bio-polymer for various applications. Des. Monomers Polym. 2009, 12, 377-404.

4. Zhong, Z.; Xing, R.; Liu, S.; Wang, L.; Cai, S.; Li, P. Synthesis of acyl thiourea derivatives of chitosan and their antimicrobial activities in vitro. Carbohydr. Res. 2008, 343, 566-570.

5. Fekry, A.M.; Mohamed, R.R. Acetyl thiourea chitosan as an eco-friendly inhibitor for mild steel in sulphuric acid medium. Electrochim. Acta 2010, 55, 1933-1939.

6. Qaqish, R.B.; Amiji, M.M. Synthesis of a fluorescent chitosan derivative and its application for the study of chitosan-mucin interactions. Carbohydr. Polym. 1999, 38, 99-107.

7. Baba, Y.; Noma, H.; Nakayama, R.; Matsushita, Y. Preparation of chitosan derivatives containing methylthiocarbamoyl and phenylthiocarbamoyl groups and their selective adsorption of copper(II) over iron(III). Anal. Sci. 2002, 18, 359-361.

8. Monier, M.; Abdel-Latif, D.A. Preparation of cross-linked magnetic chitosan-phenylthiourea resin for adsorption of $\mathrm{Hg}(\mathrm{II}), \mathrm{Cd}(\mathrm{II})$ and $\mathrm{Zn}(\mathrm{II})$ ions from aqueous solutions. J. Hazard. Mater. 2012, 209-210, 240-249.

9. Yan, M.; Gu, H.; Liu, Z.; Guo, C.; Liu, S. Effective near-infrared absorbent: ammonium tungsten bronze nanocubes. RSC Adv. 2015, 5, 967-973.

10. Guo, C.; Yin, S.; Dong, Q.; Sato, T. Simple route to $\left(\mathrm{NH}_{4}\right) x \mathrm{WO}_{3}$ nanorods for near infrared absorption. Nanoscale 2012, 4, 3394-3398. 
11. Lee, T.H.; Ryu, J.Y.; Kim T.H.; Moon, S.H.; Ahn, D.K.; Han, M.K.; Cho, E.Y.; Shon, I.S., Son, S.M. Study of NIR-dye stability in optical film for the PDP filter. Mol. Cryst. Liq. Cryst. 2009, 514, 619-631.

12. Wei, P.-R.; Chen, S.-H.; Liao, W.-N.; Kao, K.-C.; Weng, C.-F.; Lee, C.-H. Synthesis of chitosan-coated near-infrared layered double hydroxide nanoparticles for in vivo optical imaging. J. Mater. Chem. 2012, 22, 5503-5513.

13. Hayasaka, H.; Takano, T.; Satake, T. The preparation of $N$-thiocarbamoyl chitosan derivatives and their properties - The absorption of metals and the absorption of near infrared rays. Chitin Chitosan Res. 1996, 2, 182-183.

14. Shibano, M.; Nishida, S.; Saito, Y.; Kamitakahara, H.; Takano, T. Facile synthesis of acyl chitosan isothiocyanates and their application to porphyrin-appended chitosan derivative. Carbohydr. Polym. 2014, 113, 279-285.

15. Takano, T.; Shibano, M. Chitosan Isothiocyanate Derivative and Method for Producing Same. WO201318767, 15 August 2013.

16. Hu, Z.S.; Dong, J.X.; Chen, G.X. Study on antiwear and reducing friction additive of nanometer ferric oxide. Tribol. Int. 1998, 31, 355-360.

17. Cardoso, J.; Gomezdaza, O.; Ixtlilco, L.; Nair, M.T.S.; Nair, P.K. Conductive copper sulfide films on polyimide foils. Semicond. Sci. Technol. 2001, 16, 123-127.

18. Offiah, S.U.; Ugwoke, P.E.; Ekwealor, A.B.C.; Ezugwu, S.C.; Osuji, R.U.; Ezema, F.I. Structural and spectral analysis of chemical bath deposited copper sulfide thin films for solar energy conversions. Dig. J. Nanomater. Biostruct. 2012, 7, 165-773.

19. Brader, M.L.; Ainscough, E.W.; Baker, E.N.; Brondie, A.M. Copper(II) promoted desulphurization of $N$-phenylthioureas. The synthesis and X-ray structure of [Cu(bipy)(pc)2]2. Polyhedron 1989, 8, 2219-2221.

20. Inoue, K.; Yoshizuka, K.; Ohto, K.; Nakagawa, H. Solvent extraction of some metals ions with lipophilic chitosan chemically modified with functional groups of dithiocarbamate. Chem. Lett. 2001, 30, 698-699. 